\title{
THE REGULAR LOCAL NONINTERACTING CONTROL PROBLEM FOR NONLINEAR CONTROL SYSTEMS*
}

\author{
H. NIJMEIJER $\dagger$ AND J. M. SCHUMACHER $\ddagger$
}

Abstract. We study the Noninteracting Control Problem for affine nonlinear control systems under the assumption that the number of scalar inputs equals the number of vector outputs. Our purpose is to find a static state feedback law for the system which achieves noninteraction. Using the recently developed differential geometric approach to nonlinear systems theory and working under a set of regularity assumptions, we give necessary and sufficient conditions for the local solvability of the problem. This work extends earlier results in the "geometric approach" for linear systems.

Key words. nonlinear control systems, noninteracting control, controlled invariance, controllability distributions

AMS(MOS) subject classifications. 93C10, 49E05

1. Introduction. This paper is intended as a contribution to the theory of noninteracting control of nonlinear systems. In general terms, the problem can be described as follows. Suppose that a dynamical system has been given, in which two sets of variables have been designated as instruments and as targets, respectively. The targets and instruments may be either scalar variables or vectors. One says that we have a situation of noninteraction (or input-output decoupling) if each instrument affects only one target and none of the others. If the given system does not have this property, one may ask whether it is possible to add control loops to the system in such a way, that noninteraction is obtained. This is the problem of noninteracting control.

To arrive at a precise problem statement, one has to specify: (i) the class of systems under study, (ii) the precise nature of the noninteraction one wants to obtain, and (iii) the control format. In this paper, we shall consider "affine" [13], [18], [22] systems, which constitute a class of nonlinear systems that has received considerable attention. We will assume that the input variables ("instruments") are scalars, but we allow the output variables ("targets") to be vectors. The condition of noninteraction will be defined using the concepts of "controllability distributions" [11], [14] and "output controllability" [16], [17]. The control schemes we shall consider consist of locally defined state feedback and (state-dependent) precompensation. This combination is often referred to in the literature simply as "static state feedback" [8], [18] or even just "feedback". Definitions of the concepts mentioned here will be given below.

The noninteracting control problem has been studied extensively and from various points of view. Most of the literature is concerned with linear systems. In this field, one has the option of dealing with the problem via the transfer function, and this approach has been taken in some of the earliest work in noninteraction [5], [10] as well as in recent contributions [6]. Within the state-space framework, a breakthrough was made around 1970 ([24]; see also [1]). The innovation centered around the introduction of the notion of "controllability subspace" as a means of expressing the intuitive notion of "subsystem", which is of obvious importance in the theory of noninteraction. Controllability subspaces came to play a key role in a successful line of research that has been termed the "geometric approach" to linear systems theory.

\footnotetext{
* Received by the editors May 17, 1983, and in revised form July 10, 1985.

$\dagger$ Department of Applied Mathematics, Twente University of Technology, P.O. Box 217, 7500 AE Enschede, the Netherlands.

$\ddagger$ Centre for Mathematics and Computer Science, Kruislaan 413, 1098 SJ Amsterdam, the Netherlands.
} 
For more detailed accounts of the long history of noninteracting control of linear systems, we refer to [12] and [6]. In the nonlinear domain, progress has been slower. "Although efforts have been made to develop a decoupling theory for nonlinear systems, (...) considerable difficulties (...) have so far inhibited substantial progress", wrote the authors of [12] in 1971. A criterion for achievability of noninteraction by "static state feedback" was given by Sinha [20] for the case of scalar inputs and scalar outputs. Further work under the same restriction was reported in [3].

In the award winning paper [8] Isidori et al. were the first authors to give a geometric formulation for the general noninteracting control problem. They presented a solution to this problem [8, Thm. 5.1]; however, their necessary and sufficient conditions for solvability are, in the general case (allowing for vector outputs), not constructive (see [8, Thm. 5.1]). The purpose of this paper is to give constructive (verifiable) necessary and sufficient conditions also in the case of vector outputs, pertaining to the solvability of what we will call the regular local noninteracting control problem. In contrast to the results of [20], [3], [2] and also [8] we will use a nonlinear version of the concept of controllability subspaces. This extension was made in [11] and [14], leading to various definitions for the so-called "controllability distributions". The concept was applied to solve special versions of the decoupling problem in [16] and [17]. In this paper, we will show that controllability distributions can be used to rederive a major result from the linear theory [24] in a nonlinear context. This continues a line of research [7]-[9], [11], [13]-[18] that is directed towards a systematic generalization of the "geometric approach" [23] to nonlinear systems, using the methods of differential geometry.

The organization of the paper is as follows. In $\S 2$, the precise formulation is given of the decoupling problem that we consider here. The main result follows in $\S 3$, which is largely devoted to lemmas that are needed in the sufficiency part of the proof. Some remarks on the structure of a decoupled system are given in $\S 4$.

2. Problem formulation. Consider the affine nonlinear control system

$$
\dot{x}(t)=A(x(t))+\sum_{i=1}^{m} B_{i}(x(t)) u_{i}(t)
$$

where $x$ are local coordinates of a smooth $n$-dimensional manifold $M, A, B_{1}, \cdots, B_{m}$ are smooth vector fields on $M$ and $u_{i}: \mathbb{R}_{+} \rightarrow \mathbb{R}$ is a piecewise smooth input function, $i \in \underline{m}$. Together with the dynamics (2.1) we consider as output functions

$$
z_{i}(t)=C_{i}(x(t)), \quad i \in \underline{m}
$$

where $C_{i}: M \rightarrow N_{i}$ is a smooth map from $M$ to a smooth $p_{i}$-dimensional manifold $N_{i}$, $p_{i} \geqq 1, i \in \underline{m}$. We assume that each $C_{i}, i \in \underline{m}$, is a surjective submersion. We will observe later on that the output functions $C_{i}: M \rightarrow N_{i}$ play no role beyond specification of the distributions $\operatorname{Ker} C_{i *}, i \in \underline{m}$. To rule out obvious unsolvable problems, we will assume that the maps $C_{i}$ are mutually independent, i.e. the rank of the map $C=$ $\left(C_{1}, \cdots, C_{m}\right): M \rightarrow N_{1} \times \cdots \times N_{m}$ equals $p_{1}+\cdots+p_{m}$. Also we will assume-as is usual in the differential geometric approach - that the distribution generated by the input vector fields has no singularities, so

$$
\operatorname{dim} \Delta_{0}:=\operatorname{dim}\left\{B_{1}, \cdots, B_{m}\right\}=m .
$$

Furthermore we will assume that for the system (2.1) the accessibility distribution, see [14], equals $T M$. That is, the system (2.1) is strongly accessible, cf. [21], [22], i.e. the set of reachable points at time $t>0$ from $x_{0} \in M$ has a nonempty interior in $M$. In this 
paper we allow for static state feedback. Thus an admissible control law is of the form

$$
u=\alpha(x)+\beta(x) v,
$$

where $\alpha: M \rightarrow \mathbb{R}^{m}, \quad \beta: M \rightarrow \mathbb{R}^{m \times m}$ are smooth maps, and $v=\left(v_{1}, \cdots, v_{m}\right)^{t} \in$ $\mathbb{R}^{m}$ represents a new input. To keep as much open-loop control as possible, we seek $\beta$ such that $\beta(x)=\left(\beta_{i j}(x)\right)$ is nonsingular for all $x \in M$. Applying the feedback law (2.3) to $(2.1)$, we obtain as the new dynamics

$$
\dot{x}(t)=\tilde{A}(x(t))+\sum_{i=1}^{m} \tilde{B}_{i}(x(t)) v_{i}(t),
$$

where

$$
\begin{aligned}
& \tilde{A}(x)=A(x)+\sum_{i=1}^{m} B_{i}(x) \alpha_{i}(x), \\
& \tilde{B}_{i}(x)=\sum_{j=1}^{m} B_{j}(x) \beta_{j i}(x), \quad i \in \underline{m} .
\end{aligned}
$$

In the static state feedback noninteracting control problem we seek a control law (2.3) such that in the modified dynamics the control $v_{i}(\cdot)$ does not affect the outputs $z_{j}(\cdot)$ for $j \neq i$; moreover we want the scalar input $v_{i}(\cdot)$ to "control" the corresponding (vector-valued) output $z_{i}(\cdot), i \in \underline{m}$. This problem can be nicely formulated in a differential geometric framework, see also [8]. For doing so we need some terminology. Consider the set $V(M)$ of all smooth vector fields on $M$ as a Lie algebra with Lie product $\left[X_{1}, X_{2}\right]$ for $X_{1}, X_{2} \in V(M)$. For any set of vector fields $S \subset V(M)$ we denote by $\{S\}_{L A}$ the Lie subalgebra generated by $S$. Furthermore for $X_{1}, X_{2} \in V(M)$ define $a d_{X_{1}}^{0} X_{2}=X_{2}, a d_{X_{1}}^{1} X_{2}=\left[X_{1}, X_{2}\right]$ and recursively $a d_{X_{1}}^{k} X_{2}=\left[X_{1}, a d_{X_{1}}^{k-1}\right], k=2,3, \cdots$. Associated with the system (2.4) we define the following Lie algebras, see also [19]:

$$
\begin{aligned}
& L_{0}=\left\{a d_{\tilde{A}}^{k} \tilde{B}_{j}, k \in \mathbb{Z}_{+}, j \in \underline{m}\right\}_{L A}, \\
& L_{0 i}=\left\{a d_{\tilde{A}}^{k} \tilde{B}_{i}, k \in \mathbb{Z}_{+}\right\}_{L A}, \quad i \in \underline{m},
\end{aligned}
$$

and the Lie ideal generated by $L_{0 i}$ in $L_{0}$ which will be denoted by $\hat{L}_{0 i}, i \in \underline{m}$. Clearly $L_{0 i} \subset \hat{L}_{0 i}, i \in \underline{m}$. Also we introduce the corresponding distributions

$$
R_{0}=\operatorname{Span}\left\{L_{0}\right\}, \quad R_{i}=\operatorname{Span}\left\{L_{0 i}\right\}, \quad \hat{R}_{i}=\operatorname{Span}\left\{\hat{L}_{0 i}\right\}, \quad i \in \underline{m} .
$$

To take care that in the new dynamics $(2.4)$ the input $v_{i}(\cdot)$ has no interaction with $z_{j}(\cdot), j \neq i$, we must have

$$
\hat{R}_{i} \subset \bigcap_{j \neq i} \operatorname{Ker} C_{j *}, \quad i \in \underline{m} .
$$

To achieve that $v_{i}(\cdot)$ "controls" the corresponding output $z_{i}(\cdot), i \in \underline{m}$, we need the nonlinear version of output controllability, that is

$$
C_{i *} R_{i}=T N_{i}, \quad i \in \underline{m}
$$

which is equivalent to the fact that the set of reachable output values has nonempty interior in $N_{i}, i \in \underline{m}$, see [16], [17]. Because $R_{i} \subset \hat{R}_{i}$, we see that (2.9) implies

$$
C_{i *} \hat{R}_{i}=T N_{i}, \quad i \in \underline{m} \text {. }
$$

There is a nice and in our context useful interpretation of (2.8), (2.9) and (2.10) in geometric terms. Recall the following definitions, see [7], [9], [13], [18]. An involutive 
distribution $D$ on $M$ is called controlled invariant if there exists a feedback (2.3) such that the modified dynamics (2.4)-(2.5) satisfies

$$
\begin{aligned}
& {[\tilde{A}, D] \subset D,} \\
& {\left[\tilde{B}_{i}, D\right] \subset D, \quad i \in \underline{m} .}
\end{aligned}
$$

An involutive distribution $D$ on $M$ is called a (degenerate) controllability distribution if there exists a feedback (2.3) and a subset $I \subset \underline{m}$ such that

$$
D=\operatorname{Span}\left(\left\{a d_{\tilde{A}}^{k} \tilde{B}_{i} \mid i \in I, k \in \mathbb{Z}_{+}\right\}_{L A}\right) .
$$

Finally an involutive distribution $D$ on $M$ is called a regular controllability distribution if there exists a feedback (2.3) and a subset $I \subset \underline{m}$ such that

$$
\begin{aligned}
& {[\tilde{A}, D] \subset D,} \\
& {\left[\tilde{B}_{i}, D\right] \subset D, \quad i \in \underline{m}}
\end{aligned}
$$

and

$$
D=\operatorname{Span}\left(\left\{a d_{\tilde{A}}^{k} \tilde{B}_{i}, a d_{\tilde{B}_{j}}^{k} \tilde{B}_{i} \mid i \in I, k \in \mathbb{Z}_{+}\right\}_{L A}\right) .
$$

Returning to the noninteracting control problem, we see, by definition of the distributions $\hat{R}_{i}, i \in \underline{m}$, that for all $i \in \underline{m}$

$$
\begin{aligned}
& {\left[\tilde{A}, \hat{R}_{i}\right] \subset \hat{R}_{i},} \\
& {\left[\tilde{B}_{j}, \hat{R}_{i}\right] \subset \hat{R}_{i}, \quad j \in \underline{m} .}
\end{aligned}
$$

That is, $\hat{R}_{i}$ is controlled invariant and moreover it is a regular controllability distribution, $i \in \underline{m}$, whereas $R_{i}$ is a degenerate controllability distribution, $i \in m$.

In this way the static state feedback noninteracting control problem can be stated as follows:

Given the system (2.1)-(2.2), find, if possible, a feedback law (2.3) such that the distributions $\hat{R}_{i}$ defined by (2.6)-(2.7) satisfy (2.8) and (2.10).

In this paper we will solve a regular local version of this problem, to be called the regular local noninteracting control problem. In this context "local" means, that given an arbitrary initial state $x_{0} \in M$, we are interested in the existence of a local feedback (2.3), i.e. the maps $\alpha$ and $\beta$ in (2.3) are only well defined on a neighborhood of $x_{0}$. Working locally, we are able to fully exploit the differential geometric approach set up in [7], [8] and worked out in a series of papers [9], [13]-[18], [22], [25], [26]. It is a logical first step, and a common practice in the literature just cited, to exclude singularities. In this paper, too, we shall work under a series of regularity assumptions. In particular, we look for a specific set of "regular" distributions $R_{i}^{*}, i \in \underline{m}$, that satisfy the conditions (2.8) and (2.10). The exact definition of the word "regular" will be given in $\S 3$; among its implications is that we demand that the distributions $\cup_{i \in I} R_{i}^{*}$ and $\left(\cup_{i \in I} R_{i}^{*}\right) \cap \Delta_{0}$ have constant dimension for all $I \subset \underline{m}$. This surely is a restrictive assumption, but we feel that it is necessary to complete the "regular" analysis before one can hope to successfully attack the singular cases. Moreover, one should realize that for analytic systems (2.1)-(2.2) the regularity assumptions will hold on an open and dense submanifold $M^{\prime}$ of $M$. Therefore, a (global) solution to the general noninteracting control problem has to satisfy the necessary and sufficient conditions of the next section on this submanifold $M^{\prime}$. 
3. Necessary and sufficient conditions for the regular local noninteracting control problem. We now come to the necessary and sufficient conditions for the solvability of the regular local noninteracting control problem. For this we need some notation and assumptions.

A1. The distribution $\Delta_{0}=\operatorname{Span}\left\{B_{1}, \cdots, B_{m}\right\}$ has dimension $m$ on $M$.

A2. The strong accessibility distribution of (2.1), $R_{0}=\operatorname{Span}\left\{a d_{A}^{k} B_{i} \mid k \in \mathbb{Z}_{+}\right\}_{L A}$ has dimension $n$.

A3. The output maps (2.2) are mutually independent, i.e. the rank of the map $C=\left(C_{1}, \cdots, C_{m}\right): M \rightarrow N_{1} \times \cdots \times N_{m}$ equals $p_{1}+\cdots+p_{m}$.

For each subset $I \subset \underline{m}$ let us define $R_{I}^{*}$ as the maximal regular local controllability distribution contained in $\bigcap_{j \notin I} \operatorname{Ker} C_{j *}$. The involutive distributions $R_{I}^{*}, I \subset \underline{m}$, are well defined (see [11], [14]) but their dimension may vary on $M$. (We set $R_{m}^{*}=T M$, as a consequence of the rule from logic that the empty intersection of parts is the whole.)

A4. The distributions $R_{I}^{*}, \sum_{i \in I} R_{i}^{*},\left(\sum_{i \in I} R_{i}^{*}\right) \cap \Delta_{0}, I \subset \underline{m}$, as well as $\overline{\sum_{i \in I} R_{i}^{*}}$ and $\overline{\sum_{i \in I} R_{i}^{*} \cap \Delta_{0}}, I \subset \underline{m}$, all have constant dimension on $M$ (here the bar denotes involutive closure).

THEOREM 3.1. Consider the system (2.1)-(2.2) and assume A1-A4 hold. Then the regular local static state feedback noninteracting control problem is solvable if and only if

$$
\Delta_{0}=\Delta_{0} \cap R_{1}^{*}+\cdots+\Delta_{0} \cap R_{m}^{*} .
$$

Furthermore, if these conditions hold, then $\left\{R_{i}^{*}\right\}_{i=1}^{m}$ is the only solution of the noninteracting control problem.

Before we are able to prove this theorem, we need some preliminary results on the distributions $R_{I}^{*}, I \subset \underline{m}$. The following lemmas are also of independent interest, and give, even in the linear case, additional information on the structure of the distributions $R_{I}^{*}, I \subset \underline{m}$. Everywhere below, we shall consider the system (2.1)-(2.2), assuming that A1-A4 hold.

LEMMA 3.2. Suppose that (3.1) holds. Then, for all $i \in \underline{m}$,

$$
\Delta_{0} \cap R_{i}^{*} \not \subset \sum_{j \neq i} R_{j}^{*} .
$$

Proof. Suppose that (3.1) were true and that (3.2) would not hold. It would then follow that

$$
\Delta_{0} \subset \sum_{j \neq i} R_{j}^{*} \subset \overline{\sum_{j \neq i} R_{j}^{*}}
$$

Note that the last distribution in (3.3) is controlled invariant, since it is the sum of controlled invariant distributions. Under the condition of strong accessibility, any controlled invariant distribution containing $\Delta_{0}$ must be equal to TM (see [22]). Since it is clear from the definition of the distributions $R_{i}^{*}$ that

$$
\sum_{j \neq i} R_{j}^{*} \subset \overline{\sum_{j \neq i} R_{j}^{*}} \subset \operatorname{Ker} C_{i *},
$$

it would follow that $\operatorname{Ker} C_{i *}=T M$, or the map $C_{i}$ is trivial. This contradicts our assumptions.

We can use the above lemma in some counting arguments. Set $\gamma_{1}=\operatorname{dim}\left(\Delta_{0} \cap R_{1}^{*}\right)$, and define

$$
\gamma_{k}=\operatorname{dim} \sum_{i=1}^{k}\left(\Delta_{0} \cap R_{i}^{*}\right)-\operatorname{dim} \sum_{i=1}^{k-1}\left(\Delta_{0} \cap R_{i}^{*}\right)
$$


for $k=2, \cdots, m$. Note that the $\gamma_{k}$ 's are constant by A4. It is obviously true that $\gamma_{k} \geqq 0$ for all $k$, but if (3.1) holds then it follows from the above lemma that even $\gamma_{k} \geqq 1$ for $k \in \underline{m}$. For, suppose that $\gamma_{i}=0$ for some $i$; then we would have

$$
\Delta_{0} \cap R_{i}^{*} \subset \sum_{j=1}^{i-1}\left(\Delta_{0} \cap R_{j}^{*}\right) \subset \sum_{j \neq i}\left(\Delta_{0} \cap R_{j}^{*}\right),
$$

a situation which has been excluded by Lemma 3.2. On the other hand, still under the assumption that (3.1) holds, it is clear that

$$
\sum_{k=1}^{m} \gamma_{k}=\operatorname{dim} \sum_{i=1}^{m}\left(\Delta_{0} \cap R_{i}^{*}\right)=\operatorname{dim} \Delta_{0}=m .
$$

So the $\gamma_{k}$ 's form a set of $m$ integers $\geqq 1$ which add up to $m$. It follows, of course, that $\gamma_{k}=1$ for all $k$. As a consequence,

$$
\operatorname{dim} \sum_{i=1}^{j}\left(\Delta_{0} \cap R_{i}^{*}\right)=\sum_{i=1}^{j} \gamma_{j}=j
$$

for all $j \in \underline{m}$. Since the order in which we numbered the output functions is arbitrary, the conclusion can be formulated as follows.

Lemma 3.3. Suppose that (3.1) holds. Then, for all $I \subset \underline{m}$,

$$
\operatorname{dim} \sum_{i \in I}\left(\Delta_{0} \cap R_{i}^{*}\right)=|I| .
$$

An obvious consequence of this is that the distributions $\Delta_{0} \cap R_{i}^{*}$ are independent. Now, set $\theta_{1}=\operatorname{dim}\left(\Delta_{0} \cap R_{1}^{*}\right)$ and define the constants (see A4)

$$
\theta_{k}=\operatorname{dim}\left(\Delta_{0} \cap \sum_{i=1}^{k} R_{i}^{*}\right)-\operatorname{dim}\left(\Delta_{0} \cap \sum_{i=1}^{k-1} R_{i}^{*}\right)
$$

for $k=2, \cdots, m$. If we reason in the same way as above, now using the fact that $\sum_{i=1}^{m} R_{i}^{*}=T M$, then the conclusion we obtain is the following.

Lemma 3.4. Suppose that (3.1) holds. Then, for all $I \subset \underline{m}$,

$$
\operatorname{dim}\left(\Delta_{0} \cap \sum_{i \in I} R_{i}^{*}\right)=|I| \text {. }
$$

Since it is clear that

$$
\sum_{i \in I}\left(\Delta_{0} \cap R_{i}^{*}\right) \subset \Delta_{0} \cap \sum_{i \in I} R_{i}^{*}
$$

for all $I \subset \underline{m}$, we obtain as a corollary:

LemMa 3.5. Suppose that (3.1) holds. Then, for all $I \subset \underline{m}$,

$$
\Delta_{0} \cap \sum_{i \in I} R_{i}^{*}=\sum_{i \in I}\left(\Delta_{0} \cap R_{i}^{*}\right) .
$$

Set $\rho_{1}=\operatorname{dim}\left(R_{1}^{*} \cap \Delta_{0}\right)$, and define

$$
\rho_{k}=\operatorname{dim}\left(R_{\underline{k}}^{*} \cap \Delta_{0}\right)-\operatorname{dim}\left(R_{\underline{k-1}}^{*} \cap \Delta_{0}\right)
$$

for $k=2, \cdots, m$. These numbers are constants by Assumption A4. We can use them to prove:

Lemma 3.6. Suppose that (3.1) holds. Then, for all $I \subset \underline{m}$,

$$
\operatorname{dim}\left(\Delta_{0} \cap R_{I}^{*}\right)=|I| \text {. }
$$


Proof. It is sufficient to show that $\rho_{k} \neq 0$ for all $k \in \underline{m}$. So, suppose that $\rho_{k}=0$ for some $k \in \underline{m}$. Then we have

$$
R_{\underline{\underline{k}}}^{*} \cap \Delta_{0}=R_{\underline{k-1}}^{*} \cap \Delta_{0} .
$$

The distributions $R_{k-1}^{*}$ and $R_{k}^{*}$ are both regular local controllability distributions, and, of course, $R_{k-1}^{*} \subset R_{k}^{*}$. It follows from [17] that there exists a feedback $u=\alpha(x)+\beta(x) v$ for the system (2.1) such that both $R_{k}^{*}$ and $R_{k-1}^{*}$ are invariant for the modified dynamics. It then follows from the characterization of regular local controllability distributions given in [11] (Lemma 4.1) that

$$
R_{\underline{\underline{k}}}^{*}=R_{\underline{k-1}}^{*} \text {. }
$$

Since $R_{k}^{*} \subset R_{\underline{k}}^{*}$ and $R_{\underline{k-1}}^{*} \subset \operatorname{ker} C_{k *}$, we obtain

$$
R_{k}^{*} \subset \operatorname{Ker} C_{k *} .
$$

Since we also have, by definition,

$$
\sum_{i \neq k} R_{k}^{*} \subset \operatorname{Ker} C_{k *}
$$

it follows from the strong accessibility condition that the map $C_{k}$ is trivial, and we have reached a contradiction.

We can use this to establish the following lemma, which will be instrumental in proving that, under the assumption (3.1), the distribution $\sum_{i \in I} R_{i}^{*}$ is involutive.

Lemma 3.7. Suppose that (3.1) holds. Then, for any $I \subset \underline{m}$,

$$
R_{I}^{*}=\bar{R}_{I}:=\text { inv. clos. }\left[\sum_{i \in I} R_{i}^{*}\right] \text {. }
$$

Proof. Let $I \subset \underline{m}$. Since $R_{I}^{*}$ is involutive, and $R_{i}^{*} \subset R_{I}^{*}$ for all $i \in I$, we have

$$
\sum_{i \in I} R_{i}^{*} \subset \bar{R}_{I} \subset R_{I}^{*}
$$

By Lemmas 3.4 and 3.6, it follows that

$$
\Delta_{0} \cap \tilde{R}_{I}=\Delta_{0} \cap R_{I}^{*} .
$$

From [14], we know that $\bar{R}_{I}$ is a regular local controllability distribution. By the same argument as was used in the proof of Lemma 3.6, it follows that (3.20) holds.

For linear systems, the above result already implies that, under the given circumstances, the subspace corresponding to the distribution $\sum_{i \in I} R_{i}^{*}$ is equal to the maximal controllability subspace contained in the intersection of the kernels of the output mappings $C_{i}(i \in \underline{m} \backslash I)$-a result which doesn't seem to have been formulated explicitly before. In the nonlinear context, we have to worry about involutiveness. Somewhat surprisingly, it turns out that this is not a problem. The key lemma is the following.

LEMMA 3.8. Let $R_{1}$ and $R_{2}$ be regular local controllability distributions such that $\overline{R_{1}+R_{2}}$ and $\Delta_{0} \cap \overline{R_{1}+R_{2}}$ have fixed dimension, and

$$
\Delta_{0} \cap \overline{R_{1}+R_{2}}=\Delta_{0} \cap R_{1}+\Delta_{0} \cap R_{2} .
$$

Then $R_{1}+R_{2}$ is involutive (and hence-see [14]- $R_{1}+R_{2}$ is itself a regular local controllability distribution). 
Proof. Locally, we can choose sets of independent vector fields $\left\{B_{11}, \cdots, B_{1 k}\right\}$ and $\left\{B_{21}, \cdots, B_{2 l}\right\}$ such that

$$
\begin{aligned}
& \Delta_{0} \cap R_{1}=\operatorname{Span}\left\{B_{11}, \cdots, B_{1 k}\right\}, \\
& \Delta_{0} \cap R_{2}=\operatorname{Span}\left\{B_{21}, \cdots, B_{2 l}\right\} .
\end{aligned}
$$

Note that

(3.26) $\left[B_{1 i}, R_{2}\right] \subset\left(R_{2}+\Delta_{0}\right) \cap \overline{R_{1}+R_{2}}=R_{2}+\left(\Delta_{0} \cap \overline{R_{1}+R_{2}}\right)=R_{2}+\operatorname{Span}\left\{B_{11}, \cdots, B_{1 k}\right\}$ for all $i \in \underline{k}$. Using [13], we see that there exist vector fields $\tilde{B}_{11}, \cdots, \tilde{B}_{1 k}$ such that

$$
\begin{aligned}
& -\operatorname{Span}\left\{\tilde{B}_{11}, \cdots, \tilde{B}_{1 k}\right\}=\operatorname{Span}\left\{B_{11}, \cdots, B_{1 k}\right\}=\Delta_{0} \cap R_{1}, \\
& {\left[\tilde{B}_{1 i}, R_{2}\right] \subset R_{2}, \quad i \in \underline{k} .}
\end{aligned}
$$

Likewise, one can find vector fields $\tilde{B}_{21}, \cdots, \tilde{B}_{2 l}$ such that

$$
\begin{aligned}
& \operatorname{Span}\left\{\tilde{B}_{21}, \cdots, \tilde{B}_{2 l}\right\}=\operatorname{Span}\left\{B_{21}, \cdots, B_{2 l}\right\}=\Delta_{0} \cap R_{2}, \\
& {\left[\tilde{B}_{2 i}, R_{1}\right] \subset R_{1}, \quad i \in \underline{l} .}
\end{aligned}
$$

Since $\overline{R_{1}+R_{2}}$ is controlled invariant (see [14]) and $R_{1} \subset \overline{R_{1}+R_{2}}$, we can find, as in [17], a closed-loop mapping $\tilde{A}$ such that

$$
\begin{aligned}
& {\left[\tilde{A}, R_{1}\right] \subset R_{1},} \\
& {\left[\tilde{A}, \overline{R_{1}+R_{2}}\right] \subset \overline{R_{1}+R_{2}} .}
\end{aligned}
$$

Now, note that

$$
\left[\tilde{A}, R_{2}\right] \subset\left(R_{2}+\Delta_{0}\right) \cap\left(\overline{R_{1}+R_{2}}\right)=R_{2}+\operatorname{Span}\left\{\tilde{B}_{11}, \cdots, \tilde{B}_{1 k}\right\} .
$$

It follows from (3.33) and (3.28) (see [7]) that there exist functions $\gamma_{i}(i \in \underline{k})$ such that $\bar{A}=\tilde{A}+\sum_{i=1}^{k} \tilde{B}_{1 i} \gamma_{i}$ satisfies

$$
\left[\bar{A}, R_{2}\right] \subset R_{2}
$$

Using the general formula

$$
[X \cdot c, Y]=[X, Y] \cdot c-X \cdot Y(c),
$$

we find that also

$$
\begin{aligned}
& {\left[\bar{A}, R_{1}\right] \subset R_{1},} \\
& {\left[\bar{A}, \overline{R_{1}+R_{2}}\right] \subset \overline{R_{1}+R_{2}} .}
\end{aligned}
$$

By the characterization of regular local controllability distributions in [14], we know that, if we define

$$
\hat{R}_{i}=\operatorname{Span}\left\{a d^{j} \tilde{B}_{i s} \tilde{B}_{i s} \mid s \in \underline{k} \in \mathbb{Z}_{+}\right\}, \quad i=1,2
$$

then

$$
R_{i}=\text { inv.clos. } \hat{R}_{i} \text {. }
$$

Using the Jacobi identity, one can easily prove by induction that

$$
\left[a d_{\bar{A}}^{j} \tilde{B}_{1 i}, R_{2}\right] \subset R_{2}
$$

for all $j \in \mathbb{Z}_{+}$and $i \in \underline{k}$. Using (3.35) again, we find that

$$
\left[\hat{R}_{1}, R_{2}\right] \subset R_{1}+R_{2} .
$$


Another induction argument based on the Jacobi identity then shows that

$$
\left[R_{1}, R_{2}\right] \subset R_{1}+R_{2} .
$$

Hence, $R_{1}+R_{2}$ is involutive.

The result that we were after is now an easy consequence.

LEMMA 3.9. Suppose that (3.1) holds. Then, for any subset $I \subset \underline{m}$, the distribution $\sum_{i \in I} R_{i}^{*}$ is involutive.

Proof. It is sufficient to show that $\sum_{i=1}^{k} R_{i}^{*}$ is involutive for every $k \in \underline{m}$, and we do this by induction. Of course, $R_{1}^{*}$ is involutive by definition. Now assume that $\sum_{i=1}^{k-1} R_{i}^{*}$ is involutive. Then $\sum_{i=1}^{k-1} R_{i}^{*}=R_{k-1}^{*}$ according to Lemma 3.7, and so it follows that $\sum_{i=1}^{k-1} R_{i}^{*}$ is a regular local controllability distribution. Moreover, the results of Lemmas $3.7,3.6$ and 3.5 show that

$$
\Delta_{0} \cap \text { inv.clos. }\left(\sum_{i=1}^{k} R_{i}^{*}\right)=\Delta_{0} \cap\left(\sum_{i=1}^{k-1} R_{i}^{*}\right)+\Delta_{0} \cap R_{k}^{*} .
$$

An application of the preceding lemma now completes the proof.

One notes that it is now immediate that, under the assumption (3.1), we have

$$
R_{I}^{*}=\sum_{i \in I} R_{i}^{*}
$$

just as in the linear case.

Now, let us proceed to an argument that will be directly needed in the proof of the main theorem. The issue is the "compatibility" of the distributions $R_{i}^{*}$.

LEMMA 3.10. Suppose that (3.1) holds. Then, locally, there exists a basis of vector fields $\left\{\tilde{B}_{1}, \cdots, \tilde{B}_{m}\right\}$ for $\Delta_{0}$ such that

$$
\begin{aligned}
& \operatorname{Span}\left\{\tilde{B}_{i}\right\}=\Delta_{0} \cap R_{i}^{*}, \quad i \in \underline{m}, \\
& {\left[\tilde{B}_{i}^{*}, R_{j}^{*}\right] \subset R_{j}^{*}, \quad i, j \in \underline{m} .}
\end{aligned}
$$

Proof. Take $i \in \underline{m}$, and let $\hat{B}_{i}$ be a vector field such that

$$
\operatorname{Span}\left\{\hat{B}_{i}\right\}=\Delta_{0} \cap R_{i}^{*} .
$$

From the fact that the $R_{j}^{*}$ 's are controlled invariant, it follows that

$$
\left[\hat{B}_{i}, R_{j}^{*}\right] \subset R_{j}^{*}+\Delta_{0}, \quad j \in \underline{m} .
$$

Since $\hat{B}_{i} \in R_{i}^{*}$ and $R_{i}^{*}+R_{j}^{*}$ is involutive (Lemma 3.9), we also have

$$
\left[\hat{B}_{i}, R_{j}^{*}\right] \subset R_{i}^{*}+R_{j}^{*}, \quad j \in \underline{m} .
$$

Consequently, we have (using Lemma 3.5)

(3.50) $\left[\hat{B}_{i}, R_{j}^{*}\right] \subset\left(R_{j}^{*}+\Delta_{0}\right) \cap\left(R_{i}^{*}+R_{j}^{*}\right)=R_{j}^{*}+\Delta_{0} \cap\left(R_{i}^{*}+R_{j}^{*}\right)=R_{j}^{*}+\operatorname{Span}\left\{\hat{B}_{i}\right\}$.

It is also clear that

$$
\left[\hat{B}_{i}, \sum_{j \neq i} R_{j}^{*}\right] \subset \sum_{j \neq i} R_{j}^{*}+\operatorname{Span}\left\{\hat{B}_{i}\right\}
$$

From this, it follows (see [13]) that there exists a function $\beta_{i}$ such that $\tilde{B}_{i}:=\hat{B}_{i} \beta_{i}$ satisfies

$$
\left[\tilde{B}_{i}, \sum_{j \neq i} R_{j}^{*}\right] \subset \sum_{j \neq i} R_{j}^{*} \text {. }
$$

But then we also have (from (3.50) and (3.52); cf. also [18]), for $i \neq j$

$$
\left[\tilde{B}_{i}, R_{j}^{*}\right] \subset \sum_{j \neq i} R_{j}^{*} \cap\left(R_{j}^{*}+\operatorname{Span}\left\{\tilde{B}_{i}\right\}\right)=R_{j}^{*}+\left(\sum_{j \neq i} R_{j}^{*} \cap \Delta_{0} \cap R_{i}^{*}\right)=R_{j}^{*}
$$


Of course, the equality (3.53) also holds for $i=j$ since $R_{j}^{*}$ is involutive. Going through this construction for each $i \in \underline{m}$, we obtain a set of vector fields $\left\{\tilde{B}_{1}, \cdots, \tilde{B}_{m}\right\}$ which satisfies (3.46), and which is then automatically a basis for $\Delta_{0}$.

We now proceed to the proof of the main theorem.

Proof (of Theorem 3.1). For sufficiency, we assume that (3.1) holds. First of all, note that $\sum_{i=1}^{m} R_{i}^{*}$ contains $\Delta_{0}$ and is controlled invariant for the system (2.1), so that, as noted in the proof of Lemma 3.2,

$$
\sum_{i=1}^{m} R_{i}^{*}=T M
$$

Since we have, for each $i$,

$$
\sum_{j \neq i} R_{j}^{*} \subset \operatorname{Ker} C_{i *}
$$

it follows that

$$
R_{i}^{*}+\operatorname{Ker} C_{i *}=T M \quad(i \in \underline{m})
$$

i.e., (2.14) is satisfied.

Next, we have to show that there exists a local feedback of the form $u=$ $\alpha(x)+\beta(x) v$ that leaves each of the distributions $R_{i}^{*}(i \in \underline{m})$ invariant; i.e., the $R_{i}^{*}$ 's are "compatible". An appropriate $\beta:=\operatorname{diag}\left(\beta_{1}, \cdots, \beta_{m}\right)$ was already shown to exist in Lemma 3.10. Vector fields $\tilde{B}_{1}, \cdots, \tilde{B}_{m}$ can be constructed that satisfy $\left[\tilde{B}_{i}, R_{j}^{*}\right] \subset R_{j}^{*}$ $(i, j \in \underline{m})$, and there exists a unique nonsingular map $\beta: M \rightarrow \mathbb{R}^{m \times m}$ (locally defined) such that $\tilde{B}_{i}(x)=\hat{B}_{i}(x) \beta_{i}(x)$, where the vector fields $\hat{B}_{i}$ have been selected to satisfy (3.47); clearly, these vector fields are obtained from a nonsingular transformation of the original input vector fields appearing in (2.1).

We have shown in Lemma 3.9 that $\sum_{j \neq i} R_{j}^{*}$ is locally controlled invariant, so

$$
\left[A, \sum_{j \neq i} R_{j}^{*}\right] \subset \sum_{j \neq i} R_{j}^{*}+\Delta_{0}=\sum_{j \neq i} R_{j}^{*}+\operatorname{Span}\left\{\hat{B}_{i}\right\} .
$$

Also, we have

$$
\left[\tilde{B}_{i}, \sum_{j \neq i} R_{j}^{*}\right] \subset \sum_{j \neq i} R_{j}^{*} .
$$

According to [7], it follows from this that we can, locally, define a function $\alpha_{i}$ such that the vector field $A+\tilde{B}_{i} \alpha_{i}$ leaves $\sum_{j \neq i} R_{j}^{*}$ invariant:

$$
\left[A+\tilde{B}_{i} \alpha_{i}, \sum_{j \neq i} R_{j}^{*}\right] \subset \sum_{j \neq i} R_{j}^{*} .
$$

Having done this for each $i \in \underline{m}$, we next consider the vector field $A+\sum_{j=1}^{m} \tilde{B}_{j} \alpha_{j}$. The following holds:

$$
\begin{aligned}
{\left[A+\sum_{j=1}^{m} \tilde{B}_{j} \alpha_{j}, \sum_{j \neq i} R_{j}^{*}\right] } & \subset\left[A+\tilde{B}_{i} \alpha_{i}, \sum_{j \neq i} R_{j}^{*}\right]+\left[\sum_{j \neq i} \tilde{B}_{j} \alpha_{j}, \sum_{j \neq i} R_{j}^{*}\right] \\
& \subset \sum_{j \neq i} R_{j}^{*}+\sum_{j \neq i}\left[\tilde{B}_{j} \alpha_{j}, \sum_{j \neq i} R_{j}^{*}\right] \subset \sum_{j \neq i} R_{j}^{*} .
\end{aligned}
$$

This shows that the distributions $\sum_{j \neq i} R_{j}^{*}$ are compatible. Now, define

$$
\tilde{R}_{i}=\bigcap_{j \neq i} \sum_{k \neq j} R_{k}^{*}
$$


Clearly, we have $R_{i}^{*} \subset \tilde{R}_{i}$ for all $i \in \underline{m}$. It is immediate from (3.60) that

Furthermore, one has

$$
\left[A+\sum_{j=1}^{m} \tilde{B}_{j} \alpha_{j}, \tilde{R}_{i}\right] \subset \tilde{R}_{i}, \quad i \in \underline{m} .
$$

It now follows from Lemma 4.1 of [11] that $R_{i}^{*}$ is the maximal regular local controllability distribution in $\tilde{R}_{i}$, and that $R_{i}^{*}$ is also invariant under $A+\sum_{j=1}^{m} \tilde{B}_{j} \alpha_{j}$, i.e.,

$$
\left[A+\sum_{j=1}^{m} \tilde{B}_{j} \alpha_{j}, R_{i}^{*}\right] \subset R_{i}^{*}, \quad i \in \underline{m} .
$$

With the properties (3.45), (3.46) and (3.64) all fulfilled, we see that the first part of the proof is complete. To show that (3.1) is necessary, let $\left\{R_{i}\right\}_{i \in m}$ be a set of regular local controllability distributions that gives a solution of the decoupling problem (see $\S 2)$. Since

$$
\begin{aligned}
\Delta_{0}=\operatorname{Span}\left\{B_{1}, \cdots, B_{m}\right\}=\operatorname{Span}\left\{\tilde{B}_{1}, \cdots, \tilde{B}_{m}\right\} \subset & \sum_{i=1}^{m}\left(\Delta_{0} \cap R_{i}\right) \\
& \subset \sum_{i=1}^{m}\left(\Delta_{0} \cap R_{i}^{*}\right) \subset \Delta_{0},
\end{aligned}
$$

we see immediately that (3.1) must hold. Finally, it is also clear that, for any solution of the decoupling problem, we must have

$$
\Delta_{0} \cap R_{i}=\Delta_{0} \cap R_{i}^{*} .
$$

By the same argument as was used in the proof of Lemma 3.6, it follows from (3.66) that $R_{i}=R_{i}^{*}$. This completes the proof of the theorem.

Remarks. (i) In connection with the solution of the noninteracting control problem given in [8] we note the following. The distributions $\Delta_{i}=\sum_{j \neq i} R_{j}^{*}, i \in \underline{m}$, are locally compatible, i.e. there locally exists a feedback which leaves each of the $\Delta_{i}$ 's, $i \in \underline{m}$, invariant. Moreover we have that $\tilde{B}_{j} \subset \Delta_{i} \subset \operatorname{Ker} C_{i *}, j \neq i$, and for any two disjoint subsets $I, J \subset \underline{m}$ we have $\left(\bigcap_{i \in I} \Delta_{i}\right)+\left(\bigcap_{j \in J} \Delta_{j}\right)=T M$. In other words we have shown that the $\Delta_{i}$ 's, $i \in \underline{m}$, are compatible and satisfy the conditions of Theorem 5.1 of [8]; so we have produced a constructive local solution of the input-output decoupling problem. For the case of scalar outputs, such a constructive solution has already been provided in $[8, \mathrm{Thm} .5 .2]$.

(ii) In general the feedback $u=\alpha(x)+\beta(x) v$ is only locally well defined. Without any further requirements on the state space $M$ nothing can be said about the global solution of the noninteracting control problem. For instance, the question whether or not the manifold $M$ is simply connected will probably be of importance (see [9]). From [18] comes the suggestion that the holonomy group of a certain integrable connection plays a crucial role. An approach to global problems via singularity theory is advocated by C.I. Byrnes in [27], [28]. Much further work will be needed to fully develop a theory of global nonlinear decoupling.

(iii) Combining (2.10) (or (3.56)) with the result on nonlinear systems invertibility from [15], we see that each subsystem

$$
\begin{aligned}
& \dot{x}(t)=\tilde{A}(x(t))+\tilde{B}_{i}(x(t)) v_{i}(t), \quad x(0)=x_{0}, \\
& z_{i}(t)=C_{i}(x(t))
\end{aligned}
$$


is strongly invertible at $x_{0}$. In the case that each output function is 1-dimensional, so $p_{1}=\cdots=p_{m}=1$, our results coincide with the results obtained in [20], which is the nonlinear version of a result of [4], see also [8], [2].

(iv) As we also assume that $p_{1}+\cdots+p_{m}=n$ the total output map $C=$ $\left(C_{1}, \cdots, C_{m}\right)$ is a (local) diffeomorphism, we have that $\bigcap_{i \in m} \operatorname{Ker} C_{i *}=0$ and our result follows by the fact that in this case the conditions $\Delta_{0}=\Delta_{0} \cap R_{1}^{*}+\cdots+\Delta_{0} \cap R_{m}^{*}$ and $R_{i}^{*}+\operatorname{Ker} C_{i *}=T M, i \in \underline{m}$, are equivalent. By using some easy dimension arguments one can show that $R_{i}^{*}=\bigcap_{j \neq i} \operatorname{Ker} C_{j *}$ provided that the preceding condition holds, see also [16] and (3.44).

(v) The strong accessibility assumption for (2.1) is not needed. What is necessary in Theorem 3.1 is that the strongly accessible distribution (see [14], [22]) $R_{0}$ satisfies $C_{*}\left(R_{0}\right)=T\left(N_{1} \times \cdots \times N_{m}\right)$, where $C=\left(C_{1}, \cdots, C_{m}\right)$.

(vi) If the number of input channels exceeds the number of outputs, we obtain by doing similar computations as in Lemma 3.2-Lemma 3.7 that a sufficient condition for the solvability of the regular local noninteracting control problem is $\Delta_{0}=$ $\bigoplus_{i \in m} \Delta_{0} \cap R_{i}^{*}$, provided that $A_{1}-A_{4}$ hold. This serves as a starting point for a more general decoupling problem in [26].

4. The structure of a decoupled system. Next we want to discuss the (local) structure of an input-output decoupled system. We will show that as in the linear theory, see [12], our decoupled system possesses a natural local canonical form which can be built up from the distributions $R_{i}^{*}$. An alternative derivation is given in [8], and in [25] one can find a more algebraically oriented approach to the same problem. We consider a nonlinear system (2.1)-(2.2) satisfying the conditions and assumptions for the regular local noninteracting control problem, see $\S 3$. For simplicity we will take $m=2$; the general case follows by an easy induction argument. For $m=2$ consider the nested sequence of involutive distributions

$$
R_{1}^{*} \cap R_{2}^{*} \subset R_{1}^{*} \subset R_{1}^{*}+R_{2}^{*}=T M
$$

(here the last equality follows from the strong accessibility assumption). By [19] we know that around $x_{0} \in M$ there exists a coordinate system such that

$$
\begin{aligned}
& R_{1}^{*} \cap R_{2}^{*}=\operatorname{Span}\left\{\frac{\partial}{\partial x_{3}}\right\}, \\
& R_{1}^{*}=\operatorname{Span}\left\{\frac{\partial}{\partial x_{1}}, \frac{\partial}{\partial x_{3}}\right\}, \\
& R_{1}^{*}+R_{2}^{*}=\operatorname{Span}\left\{\frac{\partial}{\partial x_{1}}, \frac{\partial}{\partial x_{2}}, \frac{\partial}{\partial x_{3}}\right\}
\end{aligned}
$$

with each $x_{i}, i \in \underline{3}$, possibly being a vector.

Let us write the (locally) decoupled system as

$$
\begin{aligned}
& \dot{x}=\tilde{A}(x)+\tilde{B}_{1}(x) v_{1}+\tilde{B}_{2}(x) v_{2}, \\
& y_{1}=C_{1}(x), \\
& y_{2}=C_{2}(x) ;
\end{aligned}
$$

then we have by definition of the regular local controllability distributions $R_{1}^{*}$ and $R_{2}^{*}$ that for $i=1,2$

$$
R_{i}=\operatorname{Span}\left\{a d_{\tilde{A}}^{k} \tilde{B}_{i}, a d_{\tilde{B}_{j}}^{k} \tilde{B}_{i} \mid k \in \mathbb{Z}_{+}, j=1,2\right\}_{L A}
$$


and so

$$
\begin{aligned}
& {\left[\tilde{A}, R_{i}^{*}\right] \subset R_{i}^{*},} \\
& {\left[\tilde{B}_{j}, R_{i}^{*}\right] \subset R_{i}^{*}, \quad j=1,2 .}
\end{aligned}
$$

From (4.4) and (4.5) one can easily verify, by using the Jacobi identity extensively, that there exist sets of vector fields $\left\{X_{\alpha}\right\}_{\alpha \in I}$ and $\left\{Y_{\beta}\right\}_{\beta \in J^{\prime}}$ which span $R_{1}^{*}$ and $R_{2}^{*}$ respectively, such that

$$
\left[X_{\alpha}, Y_{\beta}\right] \in R_{1}^{*} \cap R_{2}^{*}=\operatorname{Span}\left\{\frac{\partial}{\partial x_{3}}\right\}, \quad \alpha \in I, \quad \beta \in J .
$$

Here we recognize the ideal property of $\S 2$. But (4.6) exactly implies, e.g. [19], [22], that the distribution $R_{2}^{*}$ is locally given as

$$
R_{2}^{*}=\operatorname{Span}\left\{\frac{\partial}{\partial x_{2}}, \frac{\partial}{\partial x_{3}}\right\} .
$$

Let us now see what this implies for our system. Using (4.2)-(4.7) and the fact that $R_{1}^{*} \subset \operatorname{Ker} C_{2 *}$ and $R_{2}^{*} \subset \operatorname{Ker} C_{1 *}$ we obtain that (4.3) reads in our local coordinates as

$$
\begin{aligned}
& \dot{x}_{1}=\tilde{A}_{1}\left(x_{1}\right)+\tilde{B}_{1}\left(x_{1}\right) v_{1}, \\
& \dot{x}_{2}=\tilde{A}_{2}\left(x_{2}\right)+\tilde{B}_{2}\left(x_{2}\right) v_{2}, \\
& \dot{x}_{3}=\tilde{A}_{3}\left(x_{1}, x_{2}, x_{3}\right)+\sum_{i=1}^{2} \tilde{B}_{3 i}\left(x_{1}, x_{2}, x_{3}\right) v_{i}, \\
& y_{1}=C\left(x_{1}\right), \\
& y_{2}=C\left(x_{2}\right) .
\end{aligned}
$$

This is what one might call a local canonical form for the input-output decoupled system. This is a direct generalization of the well-known linear result of [12]. In the same way one has for $m>2$ the following local canonical form:

$$
\begin{aligned}
& \dot{x}_{1}=\tilde{A}_{1}\left(x_{1}\right)+\tilde{B}_{1}\left(x_{1}\right) v_{1}, \\
& \quad \vdots \\
& \dot{x}_{m}=\tilde{A}_{m}\left(x_{m}\right)+\tilde{B}_{m}\left(x_{m}\right) v_{m}, \\
& \dot{x}_{m+1}=\tilde{A}_{m+1}\left(x_{1}, \cdots, x_{m+1}\right)+\sum_{i=1}^{m} \tilde{B}_{(m+1) i}\left(x_{1}, \cdots, x_{m+1}\right) v_{i}, \\
& y_{1}=C\left(x_{1}\right), \\
& \quad \vdots \\
& y_{m}=C\left(x_{m}\right) .
\end{aligned}
$$

In this coordinate system one has $R_{i}^{*}=\operatorname{Span}\left\{\partial / \partial x_{i}, \partial / \partial x_{m+1}\right\}, i=1, \cdots, m$. It is immediately seen from (4.9) that we have output controllability in each channel; this is an aspect that is not covered in [8].

Acknowledgment. The developments of $\S 4$ benefited from suggestions by a reviewer. 


\section{REFERENCES}

[1] G. BASILE AND G. MARRO, A state space approach to noninteracting controls, Ricerche di Automatica, 1 (1970), pp. 68-77.

[2] D. Claude, Decoupling of nonlinear systems, Syst. Contr. Lett., 1 (1982), pp. 242-248.

[3] P. E. CROUCH AND N. CARMICHAEL, Application of linear analytic systems theory to attitude control, Report to ESTEC, Applied Systems Studies, Coventry, UK, 1981.

[4] P. L. FAlB AND W. A. Wolovich, Decoupling in the design and synthesis of multivariable control systems, IEEE Trans. Automat. Contr., AC-12 (1967), pp. 651-659.

[5] H. FREEMAN, Stability and physical realizability considerations in the synthesis of multipole control systems, AIEE Trans. (Appl. Ind.) 77 (1958), pp. 1-5.

[6] M. L. J. HAUTUS AND M. HEYMANN, Linear feedback decoupling-transfer function analysis, preprint, Feb. 1980.

[7] R. M. HIRSCHORN, ( $A, B)$-Invariant distributions and disturbance decoupling of nonlinear systems, this Journal, 19 (1981), pp. 1-19.

[8] A. IsIdori, A. J. Krener, C. Gori-Giorgi AND S. MONACO, Nonlinear decoupling via feedback: a differential geometric approach, IEEE Trans. Automat. Contr. AC-26 (1981), pp. 331-345.

[9] —_ Locally $(f, g)$-invariant distributions, Syst. Contr. Lett., 1 (1981), pp. 12-15.

[10] R. J. KavanaGH, Multivariable control system synthesis, AIEE Trans. (Appl. Ind.) 77 (1958), pp. $425-429$.

[11] A. J. KRENER AND A. ISIDORI, (ad $f, G)$-Invariant and controllability distributions in feedback control of linear and nonlinear systems, Lecture Notes in Control and Information Sciences, 39, SpringerVerlag, Berlin, 1982, pp. 157-164.

[12] A. S. Morse AND W. M. WONHAM, Status of noninteracting control, IEEE Trans. Automat. Contr., AC-16 (1971), pp. 568-581.

[13] H. NiJMeIJer, Controlled invariance for affine control systems, Int. J. Contr., 34 (1981), pp. 825-833.

[14] _ Controllability distributions for nonlinear systems, Syst. Contr. Lett., 2 (1982), pp. 122-129.

[15] - Invertibility of affine nonlinear control systems: a geometric approach, Syst. Contr. Lett., 2 (1982), pp. 163-168.

[16] - Feedback decomposition of nonlinear control systems, IEEE Trans. Automat. Contr., AC-28 (1983), pp. 861-863.

[17] - The triangular decoupling problem for nonlinear control systems, Nonlinear Anal. Appl., 8 (1984), pp. 273-279.

[18] H. NIJMEIJER AND A. J. VAN DER SCHAFT, Controlled invariance for nonlinear systems, IEEE Trans. Automat. Contr., AC-27 (1982), pp. 904-914.

[19] W. RESPONDEK, On decomposition of nonlinear control systems, Syst. Contr. Lett., 1 (1982), pp. 301-308.

[20] P. K. SINHA, State feedback decoupling of nonlinear systems, IEEE Trans. Automat. Contr., AC-22 (1977), pp. 487-489.

[21] H. J. SuSSMANN AND V. JURDJEvic, Controllability of nonlinear systems, J. Differential Equations, 12 (1972), pp. 95-116.

[22] A. J. VAN DER SCHAFT, System theoretic descriptions of physical systems, Ph.D. dissertation, Univ. Groningen, the Netherlands, 1983.

[23] W. M. Wonham, Linear Multivariable Control: a Geometric Approach, Springer, New York, 1979.

[24] W. M. WONHAM AND A. S. MORSE, Decoupling and pole assignment in linear multivariable systems: A geometric approach, this Journal, 8 (1970), pp. 1-18.

[25] H. NiJMEIJER, Noninteracting control for nonlinear systems, Proc. 22nd IEEE Conference on Decision \& Control, 1983, pp. 131-133.

[26] H. NiJMEIJER AND J. M. SCHUMACHER, Zeros at infinity for affine nonlinear control systems, IEEE Trans. Automat. Contr., AC-30 (1985), pp. 566-573.

[27] C. I. BYRNES, Toward a global theory of $(f, g)$-invariant distributions with singularities, in Mathematical Theory of Networks and Systems (Proc. MTNS '83, Beer Sheva), P. A. Fuhrmann, ed., Lecture Notes in Control and Information Science 58, Springer, New York, 1984, pp. 149-165.

[28] - Feedback decoupling of rotational disturbances for spherically constrained systems, Proc. 23rd IEEE Conference on Decision and Control, Las Vegas, 1984, pp. 421-426. 